\title{
Homeostasis properties of dynamic systems as evaluation method for the functional state of children
}

\author{
O. M. Gerget \\ Institute of Cybernetic \\ National research Tomsk polytechnic university \\ Tomsk, Russia \\ gerget@tpu.ru
}

\author{
Yu. A. Cherkashina \\ Institute of Cybernetic \\ National research Tomsk polytechnic university \\ Tomsk, Russia \\ cherr999y@tpu.ru
}

\begin{abstract}
The model, criteria and the trajectory of the equilibrium balanced functioning of dynamic biological systems are described in the paper. The concepts of system kinetic and potential energy for the description of the metabolic processes in biological systems are included. Metabolic processes in the biosystem are associated with the consumption of kinetic energy to perform work (property of reactivity) and recover of potential energy (reserve capacity of the organism). System model, which reflects the energyexchange processes in the organism and allows obtaining an adequate assessment of the state of biological systems, is shown in the work. The magistral method for the analysis of biological systems in their balanced equilibrium motion is presented. Macroparameters which evaluate not only rate of state variables, but also the nature of the system stress state have been developed. Estimates characterizing the elastic properties of the organism were obtained. Comprehensive approach of functional assessment is proposed. It provides an opportunity to detect and prevent not only diseases, but also the state on the verge of norm and pathology, which is especially important for young children. An illustrative example is presented and the results are analyzed. The deterioration of the organism's homeostatic properties indicates the appearance of defects in the internal structure of the biosystem. Therefore, a signal about violation of homeostatic properties can be used for diagnostic and prognostic purposes.
\end{abstract}

Keywords - homeostasis, the organism's tension, dynamic susceptibility, magistral approach, energy-exchange approach, modeling, classification

\section{INTRODUCTION}

An important property of biosystem is the homeostatic ability to retain the indicators unchanged under conditions of random disturbances and interaction with the environment. Homeostatic properties of the system are maintained at the expense of energy-exchange processes. These processes take place inside the system, and sustained by the supply of energy from outside. Common pattern of the biosystem, without taking into account individual characteristics, allow us to understand statistical methods of research results.

Homeostasis can be used for studying the dynamic properties of any systems that based on the principles of feedback and control. In this case, some limited area defined as the homeostatic can be identified in the state space of the system. In this area, the conditions of internal self-regulation are provided, and the normal functioning of systems in a changing environment and random perturbations is observed. Homeostasis can be compared metaphorically with the property of elastic bodies restore their geometric shapes under the influence of mechanical forces. The appearance of residual effects (not saving geometric shapes) in responses to mechanical stress is a violation of the internal structure of bodies and their elastic properties.

It is worth mentioning that the homeostatic properties of the system are caused by individual characteristics, but basically have a single structural form. If $\mathrm{m}$ vitally important variables $\mathrm{xi}$ are within acceptable limits $x i m i n \leq x i \leq x i m a x(i=1, \ldots, m)$, then we have homeostasis. The intensity of life processes decreases beyond these limits, and if there are significant violations, the process is stopped. Energy-exchanged processes are characterized by constancy in the homeostasis area.

\section{BALANCED MODEL OF DYNAMIC SYSTEMS FUNCTIONING}

The change of potential energy is

$$
\Delta W_{n}(t)=\dot{X}^{T}(t) q_{n} \dot{X}(t) \Delta t
$$

The change of kinetic energy is

$$
\Delta W_{k}(t)=\dot{X}^{T}(t) q_{k} \ddot{X}(t) \Delta t
$$

Active energy losses are

$$
\Delta W_{a}(t)=\dot{X}^{T}(t) q_{a} X(t) \Delta t
$$

where $q_{n}, q_{k}, q_{a}$ are symmetric positive definite matrix, the elements of which can in general depend on the time and state variables.

The change of the total energy per unit time is equal to:

$$
\frac{d W(t)}{d t}=\dot{X}^{T}(t)\left[q_{k} \ddot{X}(t)+q_{n} \dot{X}(t)+q_{a} X(t)\right]
$$

Interest is $W(t)=$ const, when the system is close to equilibrium and provides the conditions of its normal dynamic functioning. 
Consider the dynamic system represented in Fig. 1.

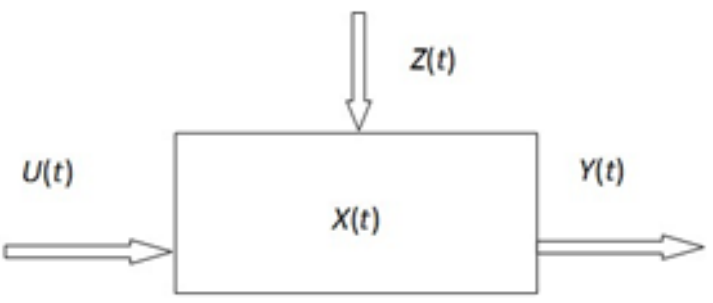

Fig. 1. General view of the system

where $\mathrm{X}(\mathrm{t})$ - vector of state variables; $\mathrm{Y}(\mathrm{t})$ - vector of system observation; $\mathrm{U}(\mathrm{t})$ - vector of external influences; $\mathrm{Z}(\mathrm{t})$ external environment.

Balanced model of a dynamic system is a stable motion of the state variables in the space near the equilibrium points. Balanced mutual change of states $\mathrm{X}(\mathrm{t})$ and external resources $\mathrm{U}(\mathrm{t})$ ensure the normal functioning of the system. This feature system of systems is actually a property of the dynamic homeostasis.

Use the equation of the system, describing its motion in state space:

$$
\dot{X}(\mathrm{t})=F(X(\mathrm{t}), U(\mathrm{t})) .
$$

For small deviations of $\mathrm{X}(\mathrm{t})$ from the equilibrium path $X_{0}(\mathrm{t})$ can be written:

$$
\Delta \dot{X}(\mathrm{t})=\left.\frac{\partial F}{\partial X}\right|_{X_{0}} \cdot \Delta X(\mathrm{t})+\frac{\partial F}{\partial U} \cdot \Delta U(\mathrm{t})
$$

Necessary resources $\Delta U(\mathrm{t})$ are determined by the deviation values of $\Delta X(\mathrm{t})$ and the matrix parameters, which characterizes the change in the system state

$$
\Delta U(\mathrm{t})=\mathrm{C}_{U} \Delta X(t)
$$

Therefore, we obtain the following expression

$$
\Delta \dot{X}(\mathrm{t})=\left[\frac{\partial F}{\partial X}+\frac{\partial F}{\partial U} \cdot \mathrm{C}_{U}\right] \Delta X(t)
$$

The rate of relative change of state variables per unit time during the operation of a dynamic system written as

$$
K_{T_{i}}=\frac{x_{i}(t+\Delta t)-x_{i}(t)}{\Delta t \cdot x_{i}(t)}=\frac{\Delta x_{i}(t)}{\Delta t \cdot x_{i}(t)} .
$$

Therefore

$$
\Delta x_{i}(t)=K_{T_{i}} \cdot x_{i}(t) \cdot \Delta \mathrm{t},
$$

or in vector form

$$
\Delta X(t)=K_{T} \cdot X(t) \cdot \Delta \mathrm{t},
$$

where $K_{T}$ - a diagonal matrix.
If we substitute the expression (4) into (3), we obtain:

$$
\Delta \dot{X}(\mathrm{t})=\left[\frac{\partial F}{\partial X}+\frac{\partial F}{\partial U} \cdot \mathrm{C}_{U}\right] \cdot K_{T} X(t) \Delta t
$$

Suppose $\Delta t \rightarrow 0$, then

$$
\lim _{\Delta t \rightarrow 0} \frac{\Delta \dot{X}(t)}{\Delta t}=\ddot{X}(t)=A_{F} K_{T} X(t)
$$

$$
\text { where } A_{F}=\left[\frac{\partial F}{\partial X}+\frac{\partial F}{\partial U} \cdot \mathrm{C}_{U}\right] .
$$

If we introduce generalized state-space variables:

$$
\begin{aligned}
& P(t)=K_{T} X(t) ; \\
& Q(t)=\dot{X}(t)
\end{aligned}
$$

Then the "(9)" can be written:

$$
\begin{aligned}
& \frac{d P(t)}{d t}=K_{T} Q(t) ; \\
& \frac{d Q(t)}{d t}=A_{F} P(t) .
\end{aligned}
$$

In the case of stable systems, these equations describe systems consisting of $\mathrm{n}$ interconnected harmonic oscillators.

The eigenvalues of the matrix $K_{T}$ and $A_{F}$ determines the frequency properties of the systems. These are basic characteristics of the functioning of the systems.

The "(11)" can be combined, if we enter one variable

$$
\begin{aligned}
& Z(t)=\{P(t), Q(t)\}=\left\{Z_{1}(t), \ldots, Z_{n}(t), \ldots Z_{2 n}\right\} . \\
& \dot{Z}(t)=M_{Z} Z(t)
\end{aligned}
$$

where

$$
M_{Z}=\left[\begin{array}{ll}
0, & K_{T} \\
A_{F}, & 0
\end{array}\right] .
$$

The described system is a self-reflection and when the matrix is a skew-symmetric, the following condition is satisfied for this system:

$$
\left(Z^{T}(t), Z(t)\right)=\|Z(t)\|^{2}=\text { const. }
$$

According to the definition square of the norm characterizes the energy of system.

Conditions skew-symmetric of matrix $M_{Z}$ is performed when $K_{T}=A_{F}$

Actually this condition determines the condition of equilibrium, balanced functioning of the system. It is valid for small deviations of nonlinear systems that are inside the area of a normally functioning homeostatic system. 


\section{PARAMETERS OF AN ORGANISM DESCRIBING THE ELASTIC PROPERTIES AS A DYNAMIC SYSTEM}

We introduce the scalar state vectors and the rate of its change (the analogue of the geometric mean index) to assess the relative change of the state variables (slow changing processes):

$$
\begin{aligned}
& r(X(t))=\sqrt[n]{\prod_{i=1}^{n} x_{i}(t)} \\
& r(\dot{X}(t))=\sqrt[n]{\prod_{i=1}^{n} \dot{x}_{i}(t)}
\end{aligned}
$$

where n- number of state variables.

The vector $r(X(t))$ is inside the segment on the pie chart:

$$
r_{\text {min }}(X(t))<r(\Delta X(t))<r_{\text {max }}(X(t)) .
$$

For small oscillations of the state vector about the equilibrium values, the following conditions are fulfilled:

$$
\frac{r(\Delta X(t))}{r\left(\mathrm{x}_{0}(t)\right)}=\frac{1}{n} \sum_{i=1}^{n} \frac{\Delta x_{i}}{x_{0 i}},
$$

where $r(\Delta X(t))=r(X(t))-r\left(X_{0}(t)\right)$.

Stressed state characteristics of the system can be determined from the relative change of indicators from the expressions:

$$
\begin{aligned}
& \alpha^{2}=\frac{\Delta X^{T}(t) \Delta X(t)}{\Delta X_{\mathcal{H}^{T}}(t) \Delta X_{\text {Д }}(t)}, \\
& \gamma^{2}=\frac{\Delta \dot{X}^{T}(t) \Delta \dot{X}(t)}{\Delta X_{0}^{T}\left(\frac{\partial F^{T}}{\partial X}\right)^{2} \Delta X_{0}} .
\end{aligned}
$$

where $\Delta X_{\not}(t)$ - the vector of acceptable deviation of state variables; $\Delta X_{0}(t)$ - the vector of equilibrium values of state variables; $\alpha^{2}$ - the coefficient characterizing the possible deviations of state variables from equilibrium values $X_{0}(t)$; $\gamma^{2}$ - the parameter determines the relative ability of regulatory mechanisms to ensure the condition of sustainable operation. The increase of $\gamma^{2}$ indicates a disruption of the system. The level of the increase makes it possible to introduce the classification of the severity of disturbances in the functioning of the systems and their quantitative evaluation.

These indicators are macroparameters characterizing the degree of tension in of the organism under normal functioning.

An important feature of the system is a dynamic sensitivity (elasticity) of the state variables, which characterizes the relative change of variables over time near the equilibrium values $X_{0}(t)$ and $\dot{X}_{0}(t)$ as:

$$
\begin{aligned}
& \left.\frac{1}{r(X(t))} \frac{d F(\mathrm{X}(t))}{d t}\right|_{X=X_{0}}=\frac{1}{n} \sum_{i=1}^{n} \frac{\dot{x}_{i}(t)}{x_{i 0}} \\
& \left.\frac{1}{r\left(\Delta \dot{X}_{0}(t)\right)} \frac{d F(\Delta \dot{X}(t))}{d t}\right|_{\dot{X}=\dot{X}_{0}}=\frac{1}{n} \sum_{i=1}^{n} \frac{\ddot{x}_{i}(t)}{\Delta \dot{x}_{i 0}(t)} .
\end{aligned}
$$

Here, the first expression indicates a possible drift of the average values of parameters from their equilibrium values. The second expression indicates the presence of cyclic processes and allows to estimate an average the dynamic characteristics of the organism.

Taking into account that:

$$
\begin{aligned}
& \dot{X}(\mathrm{t})=\mathrm{F}\left(X_{0}(\mathrm{t})\right)+\frac{\partial F^{T} X(\mathrm{t})}{\partial X} \Delta X(\mathrm{t}), \\
& \ddot{X}(\mathrm{t})=\frac{\partial F^{T} X(\mathrm{t})}{\partial X}\left[\mathrm{~F}\left(X_{0}(\mathrm{t})\right)+\frac{\partial F^{T} X(\mathrm{t})}{\partial X} \Delta X(\mathrm{t})\right],
\end{aligned}
$$

we can obtain analytical expressions for estimating the characteristics of magistral and qualitative properties of elasticity of the internal structure of the system at any time, according to the observations.

$$
\begin{gathered}
\bar{\alpha}=\frac{1}{n} \sum_{i=1}^{n} \frac{\frac{\partial F_{i}^{T}(x(\mathrm{t}))}{\partial x_{i}} \Delta x_{i}(\mathrm{t})}{x_{i_{0}}}, \\
\bar{\gamma}=\frac{1}{n} \sum_{i=1}^{n} \frac{\left(\frac{\partial F_{i}^{T}(x(\mathrm{t}))}{\partial x_{i}}\right)^{2} \Delta x_{i}(\mathrm{t})}{\Delta \dot{x}_{i_{\mu}}}
\end{gathered}
$$

The parameter $\bar{\alpha}$ characterizes the average value of state variables deviation from $X_{0}(t)$,

$\bar{\gamma}$ - determines the average value of state variables deviations of the organism dynamic interrelation that actually describes dynamic sensitivity (elasticity) of the organism.

There is a necessity to monitor changes for each variable relative to the average equilibrium state:

$$
\begin{aligned}
& \bar{\lambda}=\frac{1}{m} \sum_{j=1}^{m} \frac{\frac{\partial F^{T}\left(x_{j}(t)\right)}{\partial x_{j}} \Delta x_{j}(t)}{x_{j 0}} ; \\
& \bar{\eta}=\frac{1}{m} \sum_{j=1}^{m} \frac{\left(\frac{\partial F^{T}\left(x_{j}(t)\right)}{\partial x_{j}}\right)^{2} \Delta x_{j}(t)}{\Delta \dot{x}_{i д}(t)},
\end{aligned}
$$

where $\mathrm{m}$ is the volume of training sample for each homogeneous group.

These conditions are qualitatively characterize the elastic properties of the organism and allow us to assess the relative deviation from the equilibrium value of each variable. 
In practice, it necessary to know the numerical values of the coefficients in equations and their mutual dependence that can be obtained using the learning samples.

\section{SUMMARY OF THE RESULTS}

Consider the child's body as a kind of complex selforganizing system, interaction with the environment and has an internal energy resources. Self-organizing principle of life laid down in the human body and is realized in the process of regeneration, development and adaptation.

Learning sample is 527 objects for study. Every object is described by a state vector $\mathrm{x}=\left(\mathrm{x} \_1, \ldots, \mathrm{x} \_20\right)$. The study was carried in dynamics (15 measurements): 1, 3, 5, 7, 14, 30, 60, $90.120,150,180,240,270,300,360$ days.

Analysis of experimental data made it possible to identify patterns between the initial data and select the "essential" characteristics. The decision to use informative parameters of the cardiovascular system to develop magistral and evaluate the functional state of health in early childhood (up to 1 month) was taken.

Three groups of children were formed for research:

- «Norm» - a group of healthy children;

- «Before the crisis» - a group of children diagnosed with severity 1 ;

- «Crisis» - a group of sick children diagnosed with severity 3 or 4.

A similar gradation is considered in [1-3] for the evaluation of the critical situation in the energy sector.

Magistral trajectories of balanced functioning of the organism have been developed for all groups.

In order to identify the differences between the magistral and optimal trajectories used proximity criterion (standard deviation). Individual model trajectories based on the pattern trajectory (registered in the knowledge base).

Magistral trajectory of balanced functioning is shown in Fig. 2. Optimal trajectories for groups «Before the crisis» and «Crisis» are illustrated in Fig. 3.

We analyze the characteristics of magistrals at any time. For this purpose, we have calculated the macroparameters $\alpha$ and $\gamma$. Tension characteristic of the child's organism for each group can be given by the values of these parameters.

The average values of the tension coefficients in the first month of a child's life are presented in Table 1.

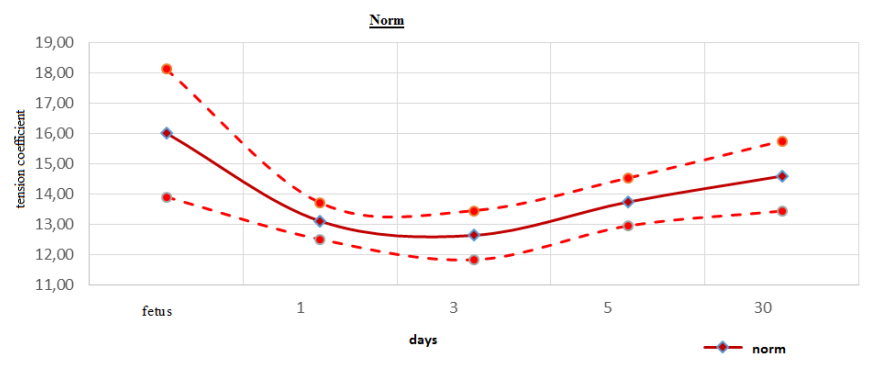

Fig.2. Magistral trajectory of balanced functioning a)

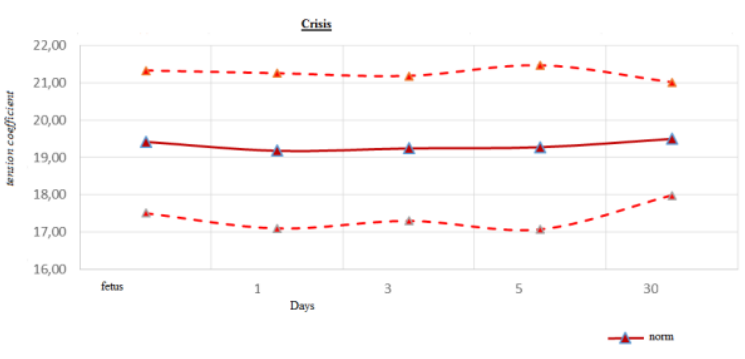

b)

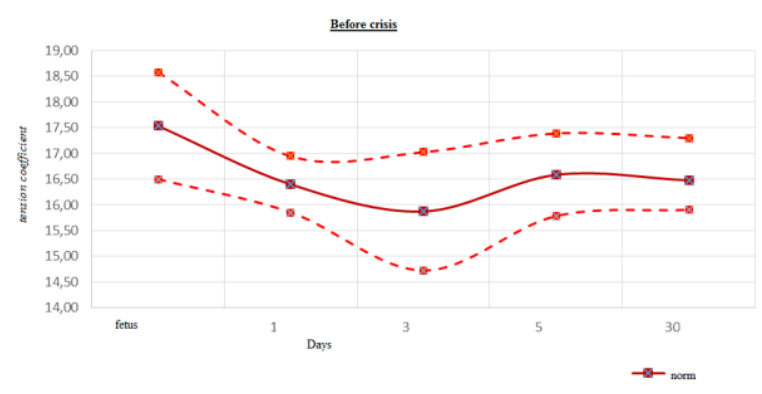

Fig. 3. Optimal trajectories a) group «Before the crisis»; b) group «Crisis»

TABLE I. THE RANGE OF THE TENSION COEFFICIENTS OF THE ORGANISM

\begin{tabular}{|c|c|c|c|c|c|c|}
\hline \multicolumn{7}{|c|}{ «Norm» } \\
\hline & fetus & 1st day & 3rd day & 7th day & 14th day & 30th day \\
\hline $\boldsymbol{\alpha}$ & $0,43 \pm 0,26$ & $0,46 \pm 0,19$ & $0,42 \pm 0,27$ & $0,4 \pm 0,16$ & $0,38 \pm 0,21$ & $0,33 \pm 0,25$ \\
\hline $\boldsymbol{\gamma}$ & $1,13 \pm 4,43$ & $0,5 \pm 0,37$ & $0,95 \pm 0,83$ & $0,6 \pm 0,41$ & $0,4 \pm 0,32$ & $0,3 \pm 0,21$ \\
\hline \multicolumn{7}{|c|}{ «Before the crisis» } \\
\hline & fetus & 1 st day & 3rd day & 7 th day & 14 th day & 30 th day \\
\hline $\boldsymbol{\alpha}$ & $0,58 \pm 0,24$ & $2,27 \pm 0,36$ & $1,79 \pm 0,33$ & $1,83 \pm 0,41$ & $1,92 \pm 0,31$ & $1,69 \pm 0,42$ \\
\hline $\boldsymbol{\gamma}$ & $0,19 \pm 1,17$ & $1,17 \pm 0,14$ & $-1,03 \pm 0,3$ & $-1,64 \pm 0,3$ & $-1,6 \pm 0,06$ & $-1,9 \pm 0,25$ \\
\hline \multicolumn{7}{|c|}{ «Crisis» } \\
\hline & fetus & 1 st day & 3 rd day & 7 th day & 14 th day & 30 th day \\
\hline $\boldsymbol{\alpha}$ & $1,05 \pm 0,63$ & $5,12 \pm 1,28$ & $3,47 \pm 0,98$ & $4,16 \pm 0,12$ & $3,82 \pm 0,83$ & $4,12 \pm 1,45$ \\
\hline $\boldsymbol{\gamma}$ & $0,82 \pm 2,56$ & $-1,4 \pm 0,02$ & $-1,5 \pm 0,12$ & $-2,4 \pm 0,02$ & $-1,4 \pm 0,02$ & - \\
& & & & & $2,14 \pm 0,05$ \\
\hline
\end{tabular}

Statistically significant differences between the samples were identified with the H Kruskal-Wallis test.

Whereas critical value $\chi_{r h}^{2}(0.05,2)=5.991$; empirical value of $\mathrm{H}$-test for the $\alpha \mathrm{H} 1=15,904$, empirical value of $\mathrm{H}$-test for the $\gamma \mathrm{H} 2=13.677$,

$$
\mathrm{H} 1>\chi_{r h}^{2}, \mathrm{H} 2>\chi_{r h}^{2},
$$

therefore the null hypothesis is rejected if $\mathrm{p} \leq 0.05$.

According to Table I, we can conclude that it is typical to have parameters $\alpha \in[0.12 ; 0.65]$ and $\gamma \in[0.05 ; 1.78]$ for healthy children. In the case where the values in the range $\alpha \in[1.27$; $2.63]$ and $\gamma \in[-2.14 ;-0.71]$ the degree of tension of the child's organism is high, so the child is in the «Before the crisis» group. To prevent the transition of the organism in a state of 
crisis it is necessary to have a medical intervention. If the value of $\alpha>3, \gamma<0$, the child's organism is in crisis.

The boundaries of macroparameters in accordance with the functioning level of the organism are presented in Table II.

TABLE II. THE GRADATION OF MACROVARIABLES

\begin{tabular}{|c|c|c|}
\hline & $\boldsymbol{\alpha}$ & $\boldsymbol{\gamma}$ \\
\hline «Norm» & $0 \leq \alpha \leq 1$ & $0 \leq \gamma \leq \infty$ \\
\hline «Before the crisis» & $1 \leq \alpha \leq 3$ & $\gamma<0$ \\
\hline «Crisis» & $3 \leq \alpha \leq 8$ & $\gamma<0$ \\
\hline
\end{tabular}

This approach allows us having observations at any time to assess the system deviation degree from the norm. Attachment of the research object to a certain group defined by a Mahalanobis metric.

The relative index of functioning has been calculated to control the dynamics of the functional state of the children's organism from different groups (Table III):

$$
\varphi_{\phi}=\frac{r(|\Delta x(t)|)}{r\left(x_{0}(t)\right)} .
$$

TABLE III. AVERAGE ESTIMATIONS

\begin{tabular}{|c|l|c|c|c|c|c|}
\hline Group & fetus & 3rd & 5th & 7th & 14th & 30th \\
\hline $\mathbf{1}$ & 0,0519 & 0,0442 & 0,0181 & 0,026 & 0,063 & 0,086 \\
\hline $\mathbf{2}$ & 0,131 & 0,2145 & 0,0813 & 0,105 & 0,243 & 0,063 \\
\hline $\mathbf{3}$ & 0,326 & 0,5629 & 0,395 & 0,318 & 0,281 & 0,3406 \\
\hline
\end{tabular}

Hidden patterns of change in the elasticity of parameters characterizing the elastic properties of the organism have been identified for a comprehensive evaluation of homeostatic properties of the organism. Results of the research for groups of «Before the crisis» and «Crisis» are shown in Tables IV and V.

TABLE IV. THE ELASTICITY OF INDICATORS FOR THE «BEFORE THE CRISIS» GROUP

\begin{tabular}{|c|l|l|l|l|l|l|l|}
\hline \multicolumn{7}{|c|}{ 《Before the crisis» } \\
\hline & fetus & $\mathbf{1 s t}$ & $\mathbf{3 r d}$ & $\mathbf{5 t h}$ & $\mathbf{7 t h}$ & $\mathbf{1 4 t h}$ & $\mathbf{3 0 t h}$ \\
\hline RR & 0,76 & 0,67 & 0,59 & 0,6 & 0,55 & 0,52 & 0,53 \\
\hline CCR & 1,3 & 1,4 & 1,48 & 1,46 & 1,47 & 1,44 & 1,47 \\
\hline dX & 0,72 & 0,7 & 0,71 & 0,66 & 0,64 & 0,62 & 0,64 \\
\hline Mo & 0,81 & 0,74 & 0,76 & 0,81 & 0,71 & 0,82 & 0,79 \\
\hline AMo & 1,1 & 1,28 & 1,33 & 1,31 & 1,1 & 1,1 & 1,27 \\
\hline INap & 1,52 & 2,31 & 2,36 & 1,7 & 1,73 & 1,69 & 1,7 \\
\hline IVR & 1,29 & 1,83 & 1,91 & 2,02 & 1,88 & 1,95 & 2,01 \\
\hline PAPR & 1,32 & 1,73 & 1,79 & 1,65 & 1,44 & 1,44 & 1,41 \\
\hline VPR & 1,41 & 1,92 & 1,92 & 1,9 & 2,17 & 2,11 & 2,19 \\
\hline Sigma & 1,04 & 1,11 & 1,13 & 0,92 & 1,04 & 0,98 & 1,02 \\
\hline NSR & 1,05 & 1,11 & 1,17 & 1,31 & 0,98 & 1,15 & 1,13 \\
\hline
\end{tabular}

TABLE V. THE ELASTICITY OF INDICATORS FOR THE «CRISIS» GROUP

\begin{tabular}{|c|c|c|c|c|c|c|c|}
\hline \multicolumn{8}{|c|}{$\begin{array}{c}\text { «Crisis» } \\
\bar{\lambda}\end{array}$} \\
\hline & fetus & $1 s t$ & $3 r d$ & 5th & $7 t h$ & 14th & 30th \\
\hline RR & 0,74 & 0,45 & 0,4 & 0,38 & 0,33 & 0,29 & 0,29 \\
\hline CCR & 1,41 & 1,74 & 1,86 & 1,87 & 1,87 & 1,82 & 1,78 \\
\hline $\mathrm{dX}$ & 0,7 & 1,36 & 0,71 & 1,14 & 0,84 & 0,62 & 0,66 \\
\hline Mo & 0,79 & 0,44 & 0,54 & 0,68 & 0,58 & 0,61 & 0,6 \\
\hline AMo & 1,26 & 1,65 & 1,72 & 1,49 & 1,5 & 1,52 & 1,4 \\
\hline INap & 1,92 & 4,59 & 4,58 & 3,44 & 3,49 & 3,61 & 3,59 \\
\hline IVR & 1,61 & 2,65 & 3,22 & 2,73 & 2,81 & 2,94 & 3,26 \\
\hline PAPR & 1,57 & 2,6 & 2,73 & 2,72 & 1,94 & 2,02 & 1,85 \\
\hline VPR & 1,62 & 2,48 & 2,91 & 3,28 & 3,02 & 3,11 & 3,37 \\
\hline Sigma & 2,48 & 1,2 & 1,31 & 1,14 & 1,04 & 1,29 & 1,43 \\
\hline NSR & 1,21 & 1,32 & 1,3 & 1,15 & 0,94 & 1,09 & 0,73 \\
\hline
\end{tabular}

Preventive maintenance for not only the disease but also the state on the verge of norm and pathology $[2,3]$ is possible with such a comprehensive approach. This approach is especially important for early childhood, when the slightest changes have a huge impact on the health of newborns.

\section{CONCLUSION}

Macroparameters that evaluate not only rate of state variables, but also the nature of the system stress state has been developed. Estimates characterizing the elastic properties of the organism were obtained. Magistral approach allows us to evaluate the deviation degree of the system from the norm. It enables prove the choice of parameters that characterize not only a magistral, but and the properties of the surrounding paths. The possibility of using the magistral technologies for monitoring dynamic systems have been demonstrated. group behavior patterns of research objects have been identified and evaluations of individual states have been obtained based on energy-exchange processes.

\section{REFERENCES}

[1] Fogel L.J., Owens A.J., Walsh M.J. Artificial intelligence through simulated evolution. - New York: John Wiley \& Sons, 1966. $-231 \mathrm{p}$.

[2] Survey of Multiobjective Evolutionary Algorithms for Data Mining. Pt. 1 / A. Mukhopadh-yay, U. Maulik, S. Bandyopadhyay, C.A. Coello // IEEE Transactions on Evolutionary Com-putation. - 2014. - Vol. 18, № 1. - P. 4-19.

[3] Survey of Multiobjective Evolutionary Algorithms for Data Mining. Pt. II // A. Mukhopadh-yay, U. Maulik, S. Bandyopadhyay, C.A. Coello // IEEE Transactions on Evolutionary Com-putation. - 2014. - Vol. 18, № 1. - P. 20-35.

[4] Carreno J.E. Multi-objective optimization by using evolutionary algorithms: The p-Optimality Criteria // IEEE Transactions on Evolutionary Computation. - 2014. - Vol. 18, № 2. - P. 167-179.

[5] Das S., Suganthan P.N. Differential Evolution: A Survey of the State-of-the-Art // IEEE Transactions on Evolutionary Computation. - 2011. - Vol. 15, № 1. - P. 4-31.

[6] Metropolis N., Ulam S. The Monte Carlo Method // Journal of the American Statistical Asso-ciation. - 1949. - Vol. 44, № 247 